\title{
Specific motor cortex hypoexcitability and hypoactivation in COPD patients with peripheral muscle weakness
}

\author{
Francois Alexandre ${ }^{1,2^{*}}$ (D) Nelly Héraud ${ }^{1}$, Emilie Tremey ${ }^{1}$, Nicolas Oliver ${ }^{1}$, Dominique Bourgouin ${ }^{1}$ and Alain Varray ${ }^{2}$
}

\begin{abstract}
Background: Peripheral muscle weakness can be caused by both peripheral muscle and neural alterations. Although peripheral alterations cannot totally explain peripheral muscle weakness in COPD, the existence of an activation deficit remains controversial. The heterogeneity of muscle weakness (between 32 and 57\% of COPD patients) is generally not controlled in studies and could explain this discrepancy. This study aimed to specifically compare voluntary and stimulated activation levels in COPD patients with and without muscle weakness.

Methods: Twenty-two patients with quadriceps weakness (COPD $\left.\mathrm{MW}_{\mathrm{W}}\right), 18$ patients with preserved quadriceps strength $\left(\mathrm{COPD}_{\mathrm{Nomw}}\right)$ and 20 controls were recruited. Voluntary activation was measured through peripheral nerve (VA $\left.A_{\text {peripheral }}\right)$ and transcranial magnetic (VA cortical $)$ stimulation. Corticospinal and spinal excitability (MEP/Mmax and Hmax/Mmax) and corticospinal inhibition (silent period duration) were assessed during maximal voluntary quadriceps contractions.

Results: COPD $\mathrm{MW}_{\text {w }}$ exhibited lower VA $A_{\text {cortical }}$ and lower MEP/Mmax compared with COPD NoMw $(p<0.05)$. Hmax/Mmax was not significantly different between groups $(p=0.25)$. Silent period duration was longer in the two groups of COPD patients compared with controls $(p<0.01)$. Interestingly, there were no significant differences between all COPD patients taken together and controls regarding $V A_{\text {cortical }}$ and MEP/Mmax.

Conclusions: COPD patients with muscle weakness have reduced voluntary activation without altered spinal excitability. Corticospinal inhibition is higher in COPD regardless of muscle weakness. Therefore, reduced cortical excitability and a voluntary activation deficit from the motor cortex are the most likely cortical mechanisms implicated in COPD muscle weakness. The mechanisms responsible for cortical impairment and possible therapeutic interventions need to be addressed.
\end{abstract}

Keywords: Chronic obstructive pulmonary disease, Motor cortex, Peripheral muscle weakness, Corticospinal excitability

\section{Background}

Central (respiratory) and peripheral (limb) muscle weakness is one of the main systemic effects of chronic obstructive pulmonary disease (COPD) [1]. It primarily affects the lower limb muscles [2], contributes to exercise intolerance [3] and is associated with increasing disability and mortality $[4,5]$.

\footnotetext{
* Correspondence: alexandre.francois88@gmail.com

'Les Cliniques du Souffle, Research Department, Groupe 5 Santé, 800 avenue Joseph Vallot, 34700 Lodève, France

${ }^{2}$ Euromov Laboratory, University of Montpellier, Montpellier, France
}

Peripheral muscle weakness can be caused by both peripheral (muscle mass and contractile properties) and neural alterations [6,7]. Several studies have indicated that peripheral mechanisms do not account for all the strength loss in COPD. For example, lower quadriceps strength per unit of muscle cross-sectional area was found despite normal contractile properties in COPD patients [8]. In addition, lower quadriceps strength is not observed in some patients when peripheral nerve stimulation is used instead of voluntary contraction $[9,10]$. This raises the hypothesis of altered neural drive to the muscle in COPD.

(c) The Author(s). 2019 Open Access This article is distributed under the terms of the Creative Commons Attribution 4.0 International License (http://creativecommons.org/licenses/by/4.0/), which permits unrestricted use, distribution, and 
Surprisingly, the validation of this hypothesis remains quite controversial. By using the twitch interpolation method, most studies have failed to observe an activation deficit in COPD during maximal voluntary contractions [11-13], while others have clearly shown this deficit during submaximal voluntary contractions [14]. By using a different method and applying sensors directly to the scalp, lower neural drive from the motor cortex was reported during maximal and submaximal voluntary contractions in COPD [15]. Both methodological concerns and the heterogeneity of muscle weakness in COPD could explain these discrepancies. First, the twitch interpolation method may be biased during maximal voluntary contractions, as the relationship between voluntary strength and the twitch-like increment in strength is no longer linear at high intensities [16-18]. The measurement of voluntary activation by motor cortex stimulation could help to resolve this issue [19]. Second, it is well known that muscle weakness is not ever-present in COPD patients since its prevalence is between 32 and 57\% $[20,21]$. None of the aforementioned studies discriminated the patients with and without muscle weakness. Thus, different proportions of COPD patients with versus without muscle weakness, depending on the study, could have biased the overall results.

Furthermore, lower neural drive to the muscle can be ascribed to at least three mechanisms, which have never been questioned in the context of muscle weakness in COPD. First, decreased excitation from the brain is strongly expected in these patients given the decreased gray matter density in the motor and prefrontal cortex $[22,23]$ and the presence of white matter lesions in the pyramidal neurons [24]. Second, lengthened latency and lower amplitude of the maximal compound muscle action potential (Mmax) have also been described in COPD patients and suggest impaired neuromuscular transmission at the motor neuron and/or the motor plate level [25-28]. Third, higher supraspinal inhibition during voluntary contraction could contribute to lowering the motor output by inhibiting the neural drive in COPD. Indeed, inflammatory mediators as well as lactate and protons [29] induce increased activity in group III or IV muscle afferents, which acts supraspinally to limit motor cortical output [30]. As COPD patients are known to exhibit high levels of chronic systemic inflammation [31] and a predominance of type II glycolytic fibers leading to elevated muscle glycolytic activity [32], the hypothesis of a motor output decrease due to increased inhibition is relevant.

The aim of the study was to address the question of a specific activation deficit in COPD with muscle weakness, and if so, by which mechanisms. We hypothesized lower motor cortex activation and excitability, higher corticospinal inhibition, and lower Mmax amplitude in patients with COPD and muscle weakness, as compared with COPD patients with normal muscle strength.

\section{Methods \\ Participants}

Forty COPD patients and 22 healthy controls, aged between 40 and 80 years, were recruited for the study (Fig. 1). The COPD patients were recruited and tested at their entrance in two French pulmonary rehabilitation centers (Cliniques du Souffle La Vallonie, Lodève, and Les Clarines, Riom-ès-Montagne, France) between 2012 and 2014. The healthy controls were recruited through an ad in a local newspaper within the same period. The participation criteria for the COPD patients were a diagnosis of COPD with forced expiratory volume in the 1st second $\left(\mathrm{FEV}_{1}\right)$ between 30 and $80 \%$ of the theoretical values (GOLD 2 and 3), with no exacerbation or weight loss in the month preceding the study. The noninclusion criteria were the same for patients and controls: inability to give written consent, inability to perform the experimental maneuvers, impaired visual function, use of drugs known to impact brain function (GABA agonist, Z-drugs, tricyclic antidepressants, melatoninergic antidepressants, selective serotonin/noradrenalin reuptake inhibitors and opioid receptor agonists), chronic current or past alcohol abuse ( $>14$ units of alcohol per week), mental disorder and neurologic or neuromuscular disease. For the diagnosis of peripheral muscle weakness, the isometric maximal quadriceps torque (QMVC) of each participant was expressed as a percentage of predicted values obtained from the national isometric muscle strength database consortium [33]. Patients with QMVC below $80 \%$ of predicted values were then assigned to the muscle weakness group $\left(\mathrm{COPD}_{\mathrm{MW}}\right)$ and the others to the non-muscle weakness group $\left(\mathrm{COPD}_{\mathrm{NoMw}}\right)$ [34]. Healthy controls with peripheral muscle weakness were excluded from the analyses $(n=2)$. All participants gave written consent. Procedures were approved by the local ethics committee (CPP SudEst VI, Clermont-Ferrand, number AU980) and complied with the principles of the Declaration of Helsinki for human experimentation.

\section{Pulmonary function tests Plethysmography}

Both patients and controls underwent plethysmography (V6200 Autobox, Sensormedics Corp., Yorba Linda, CA, USA). Measurements included forced vital capacity (FVC) and $\mathrm{FEV}_{1}$. The presence of persistent airflow obstruction and thus COPD was defined by a postbronchodilatator $\mathrm{FEV}_{1} / \mathrm{FVC}$ ratio $<0.7$ [35]. The $\mathrm{FEV}_{1}$ values were expressed as a percentage of predicted value [36]. 


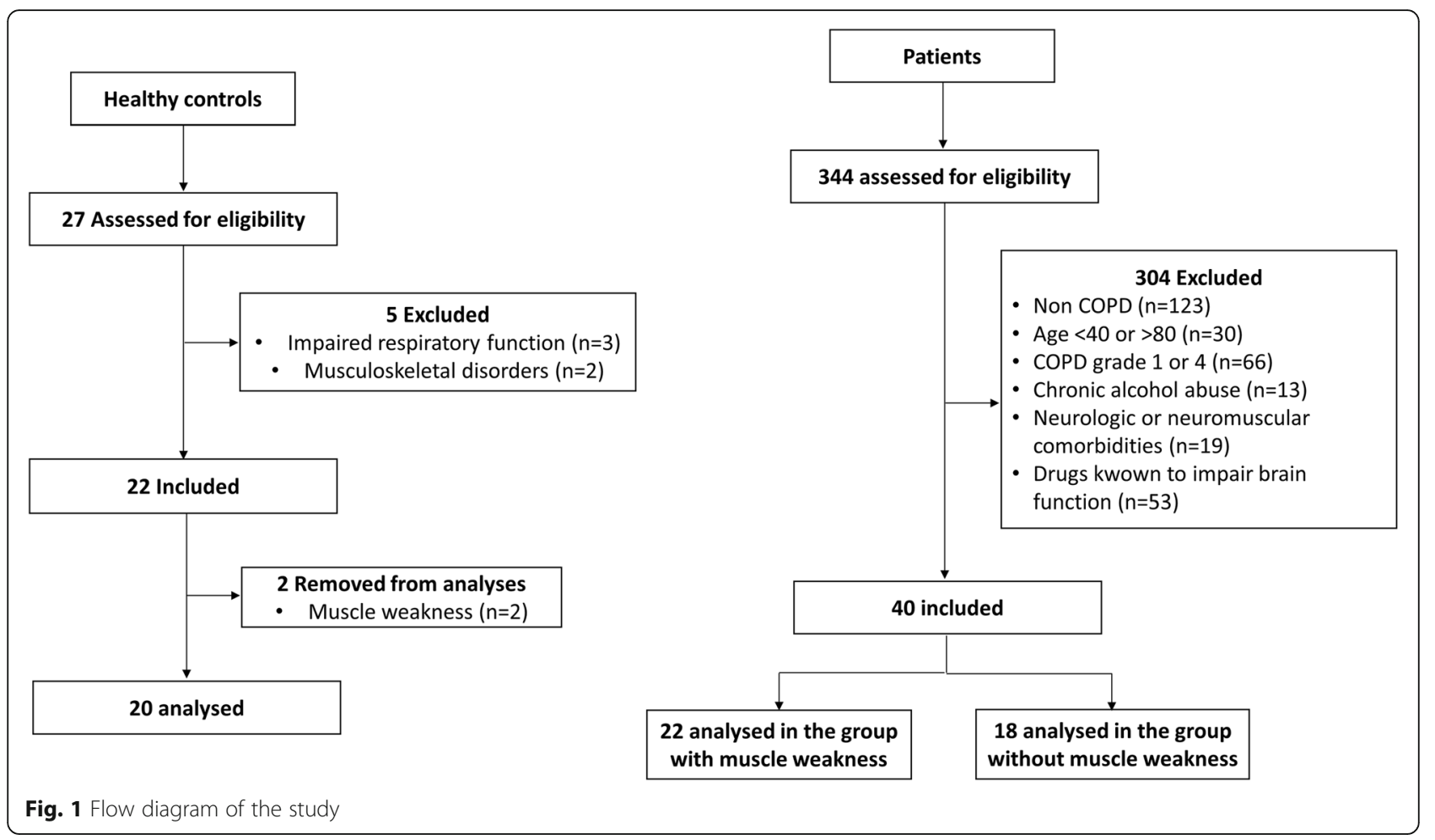

\section{Blood gas analyses}

Measurement of blood gases $\left(\mathrm{PaO}_{2}\right.$ and $\left.\mathrm{PaCO}_{2}\right)$ collected from the radial artery was performed in resting patients while they breathed room air, using a blood gas analyzer (ABL 825, Radiometer Medical, Bronshoj, Denmark).

\section{Neuromuscular tests}

\section{Experimental design}

After determination of the dominant leg [37], the participants were comfortably seated on a dedicated ergometer for knee extensor testing (Quadriergoforme, Aleo Industrie, Salome, France) equipped with a strain gauge torque sensor (Captels, Saint Mathieu de Treviers, France). The hip and the knee angle were set at $90^{\circ}$. The pelvis and the proximal extremity of the patella were securely attached to the chair in order to minimize movements of adjacent muscles. All the experimental manoeuvers of the protocol were done on the ergometer and in the same body position (including stimulations at rest). The participants were systematically familiarized to the experimental procedures the day before the protocol through a physical training session. This session included transcranial magnetic and femoral nerve stimulation recruitment curves, followed by 3 maximal voluntary contractions and several submaximal voluntary contractions at 30 and $50 \%$ of MVC lasting $5 \mathrm{~s}$ or until the targets were correctly reached, with superimposed transcranial magnetic and femoral nerve stimulations.

\section{Evaluation of isometric maximal quadriceps torque}

Isometric maximal quadriceps torque of the dominant leg was assessed as the highest torque value recorded during the protocol. Participants were verbally encouraged during each contraction to ensure maximal personal implication. QMVC was expressed in $\mathrm{Nm}$ and as a percentage of predicted values obtained from the national isometric muscle strength database consortium [33]. The maximal electrically evoked torque (quadriceps peak twitch, QPt) was assessed at rest as the highest twitch response induced by $\mathrm{I}_{\mathrm{Mmax}}$ femoral nerve stimulation $\left(\mathrm{I}_{\mathrm{Mmax}}\right.$ determination is described in the following paragraph).

\section{Evaluation of peripheral and spinal excitability by femoral nerve stimulation}

The femoral nerve stimulation was applied to assess peripheral and spinal excitability. A constant-current and high voltage stimulator (DS7AH, Digitimer, Hertforshire, UK) was used. Rectangular monophasic pulses of $500 \mu \mathrm{s}$ were used to ensure optimal activation of deeper muscle fibers [38] and to enable the appearance of $\mathrm{H}$-waves [39]. The anode, a self-adhesive electrode $(10 \times 5 \mathrm{~cm})$, was placed over the greater trochanter. The cathode, a ball electrode covered with damp foam, was placed over the participant's femoral triangle (Scarpa), 3 to $5 \mathrm{~cm}$ below the inguinal ligament. To determine optimal location, the cathode was moved by small amounts while delivering pulses at $50 \mathrm{~mA}$ until the highest $\mathrm{M}$-wave 
response was obtained over the vastus medialis with the smallest possible response over the antagonist biceps femoris. Then markers were set over the participant to maintain the cathode position. A recruitment curve was performed at rest to determine the intensities at which the highest M-wave (Mmax) and H-reflex (Hmax) were obtained. One pulse was delivered on the femoral nerve every $10 \mathrm{~s}$, with the intensity beginning at $50 \mathrm{~mA}$ and increasing by $10 \mathrm{~mA}$ until no further increase in twitch mechanical response and $\mathrm{M}$-wave amplitude occurred. The intensity used during the protocol was set as $10 \%$ above the intensity at which Mmax was elicited (supramaximal intensity noted $\mathrm{I}_{\mathrm{Mmax}}$ ). $\mathrm{I}_{\mathrm{Mmax}}$ was used to evoke M-wave at rest (Mmax) and during maximal voluntary contraction to deliver double twitch pulses (doublet) at $100 \mathrm{~Hz}$. Subsequently to the $\mathrm{I}_{\mathrm{Mmax}}$ determination, the intensity at which the maximum Hmax was obtained was carefully sought. This intensity was used to evoke Hreflex at rest (Hmax). Peripheral and spinal excitability were defined as the highest Mmax and Hmax recorded during the protocol, respectively. Hmax was normalized with respect to $\mathrm{Mmax}(\mathrm{Hmax} / \mathrm{Mmax})$ to avoid potential bias due to peripheral excitability differences. Mmax and Hmax latencies were defined as the time between the stimulation onset and the evoked potential onset.

\section{Evaluation of corticospinal excitability by transcranial magnetic stimulation}

Single transcranial magnetic stimulation (TMS) pulses of 1-ms duration were delivered over the motor cortex using a Magstim 200 (Magstim Co., Whitland, UK). During the settings, TMS pulses were delivered during isometric submaximal voluntary contraction at $10 \%$ of the maximal quadriceps torque (facilitation). The figureof-eight coil was held over the contralateral motor cortex at the optimum scalp position to elicit motor evoked potential (MEP) responses in the contralateral vastus medialis muscle. The contralateral motor cortex was first localized using the 10-10 EEG system (C3 point for right limb stimulation, $\mathrm{C} 4$ point for left limb stimulation). Then, the coil was moved by small amounts until the highest MEP response on the vastus medialis was obtained with suprathreshold stimuli, with the smallest possible response over the antagonist biceps femoris, in order to determine the optimal coil location. If significant activation of the antagonist biceps femoris muscle was noted, the coil was slightly moved, until its activation was minimized. Then markers were positioned over the participant and over the coil to maintain the coil location. After that, a recruitment curve was performed during voluntary contraction, at $10 \%$ of the maximal quadriceps torque, in order to determine the maximal intensity (noted $\mathrm{I}_{\mathrm{Mep}}$ ) [40]. One pulse was delivered every $10 \mathrm{~s}$ with increasing intensity in steps of $2 \%$ until the highest response was obtained. At least three pulses were delivered at each intensity level to check for reproducibility. The maximal intensity was defined as the intensity at which the highest MEP amplitude was obtained over the vastus medialis. This was then used during the protocol to elicit MEP responses during maximal voluntary contractions in order to assess corticospinal excitability and primary motor cortex activation. If a participant reached the maximum stimulator output without evidence of maximal MEP response (i.e., no evidence of plateau of the MEP amplitude before reaching the maximal output), the data were excluded from the analyses.

Corticospinal excitability was assessed during maximal voluntary contractions by the highest amplitude of the MEP induced by $\mathrm{I}_{\mathrm{MEP}}$ with respect to peripheral excitability (MEP/Mmax). The silent period duration was measured as the time between the MEP onset and the return of voluntary EMG activity. The central motor conduction time was calculated from the delay between stimulus artifact and the MEP onset.

\section{Evaluation of voluntary activation with femoral nerve and transcranial magnetic stimulation}

The voluntary activation was assessed by peripheral nerve stimulation $\left(\mathrm{VA}_{\text {peripheral }}\right)$ and transcranial magnetic stimulation ( $\left.\mathrm{VA}_{\text {cortical }}\right)$.

$\mathrm{VA}_{\text {peripheral }}$ was calculated according to the twitch interpolation technique (4). A supramaximal doublet was delivered during the force plateau of the maximal voluntary contraction (superimposed doublet) and $2 \mathrm{~s}$ after relaxation (control doublet). $\mathrm{VA}_{\text {peripheral }}$ was calculated as the ratio between the twitch-like increment in torque induced by the supramaximal doublet during maximal voluntary contraction and after relaxation:

$\mathrm{VA}_{\text {cortical }}(\%)=[1-($ superimposed twitch/estimated resting twitch $)] \times 100$

$\mathrm{VA}_{\text {cortical }}$ was calculated by stimulating the motor cortex during the quadriceps contractions according to the method described by Sidhu et al. [19]. The estimated resting twitch was calculated from the curve-response relationship obtained by plotting the twitch-like increment in torque induced by the transcranial magnetic pulses delivered during the last two maximal voluntary contractions, as well as those obtained during submaximal voluntary contractions at 30 and $50 \%$ of QMVC. When no linear relationship could be obtained between the voluntary force and the twitch-like increment in torque $(r<0.9)$, the data were excluded from the analyses [41]. $\mathrm{VA}_{\text {cortical }}$ was calculated as the ratio between the highest twitch-like increment in torque induced by the TMS pulses during maximal voluntary contractions and the estimated resting twitch: 
$\mathrm{VA}_{\text {cortical }}(\%)=[1-($ superimposed twitch/estimated resting twitch $)] \times 100$

\section{EMG activity}

The surface EMG activity of the vastus medialis, rectus femoris and biceps femoris was recorded throughout the protocol with Biopac technology (Biopac MP100, Biopac Systems, Santa Barbara, CA, USA). Bipolar, silver chloride, square surface electrodes with a 9-mm diameter were used (Contrôle Graphique Médical, Brie-CompteRobert, France). In order to minimize impedance $(<5$ $\mathrm{k} \Omega$ ), the skin was shaved, abraded, and cleaned with alcohol. Two electrodes were set at the middle belly of the vastus medialis, rectus femoris and long head of the biceps femoris muscles of the dominant leg with an interelectrode distance of $2 \mathrm{~cm}$. The reference electrode was placed on the opposite patella. The EMG signal was band-pass-filtered $(10-500 \mathrm{~Hz})$, amplified $(\times 1000)$ and recorded at a sample frequency of $4096 \mathrm{~Hz}$.

\section{Protocol}

The participants performed four maximal voluntary contractions of the knee extensors, each separated by $2 \mathrm{~min}$ of recovery (Fig. 2). They were asked to maintain maximal effort for at least $4 \mathrm{~s}$. During the first two maximal voluntary contraction maneuvers, a double pulse at 100 $\mathrm{Hz}$ was delivered over the femoral nerve (superimposed doublet) during the force plateau and $2 \mathrm{~s}$ after relaxation (control doublet). During the last two maximal voluntary contraction maneuvers, a single TMS pulse at $\mathrm{I}_{\text {Mep }}$ was delivered over the motor cortex to elicit MEPs during the force plateau. Three single pulses at $\mathrm{I}_{\mathrm{Mmax}}$ or Hmax intensity separated by $10 \mathrm{~s}$ were delivered twice between maximal voluntary contractions to elicit Mmax and Hmax at rest, respectively. The time interval between Mmax and Hmax stimuli was between 30 and 40s. If any pre-stimulus voluntary activity was observed, the involved stimuli were discarded. After the maximal voluntary contractions, three submaximal voluntary contractions (SVC) with visual feedback were performed at 50 and $30 \%$ of QMVC. A single TMS pulse at $\mathrm{I}_{\text {Mep }}$ was delivered during the force plateau of each SVC to elicit superimposed twitch responses at 30 and $50 \%$ of QMVC.

\section{Statistical analyses}

All statistical analyses except slope comparisons were performed using Statistica software (StatSoft, Inc., version 6.0, Tulsa, OK, USA). All data were examined for normality using a Shapiro-Wilk test. Differences between the pooled COPD patients and the healthy controls were studied using unpaired t-tests for parametric data and the non-parametric Mann-Whitney $U$ test otherwise. Differences between the $\mathrm{COPD}_{\mathrm{MW}}$ and $\mathrm{COPD}_{\mathrm{NoMW}}$ groups and healthy controls were tested using a one-way between-subject analysis of variance (ANOVA), unless when no data were available for healthy controls (i.e. blood gas analyses and comorbidities), with the 2 groups of patients compared using unpaired t-test instead. The underlying assumptions of ANOVA were checked using a Levene test (homogeneity of the variance). When the ANOVA $\mathrm{F}$ ratio was significant $(p<0.05)$, the means were compared by a Studentized Newman-Keuls (SNK) post-hoc test. Analysis of covariance (ANCOVA) was used with 1) QMVC as the criterion variable and QPt as the covariate (adjusted maximal voluntary strength), 2)

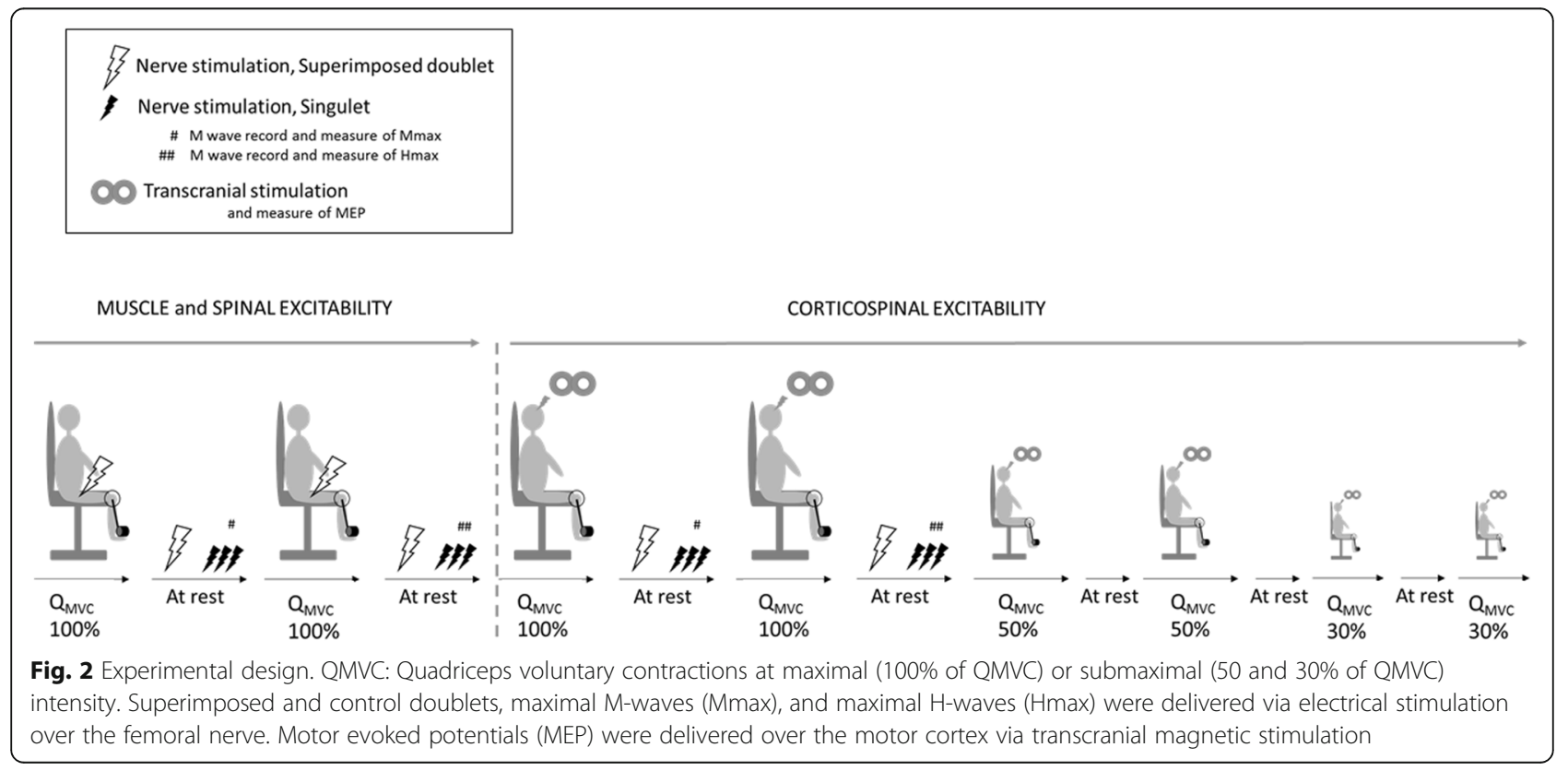


$\mathrm{VA}_{\text {cortical }}$ as the criterion variable and $\mathrm{PaO}_{2}$ as the covariate. Bivariate regression analyses were performed using the Pearson coefficient. The slopes and Y-intercepts of the relationships between QMVC and QPt were compared for differences between the three groups using a specific ANOVA procedure of the Statgraphics Centurion XVII statistical package. Data are presented mean \pm standard error (SE).

\section{Results}

\section{Controls and the pooled COPD patients}

The results from the COPD patients taken as a whole and the controls are indicative and are provided in Table 1. COPD patients presented significantly lower QMVC $\left(\mathrm{t}_{58}=3.42, p<0.05\right)$ and QPt $\left(\mathrm{t}_{57}=2.31, p<0.05\right)$

Table 1 Comparison between healthy controls and patients with COPD as a whole

\begin{tabular}{|c|c|c|c|}
\hline & Controls $n=20$ & $\operatorname{COPD} n=40$ & $p$-value ( $t$-test) \\
\hline \multicolumn{4}{|c|}{ Anthropometric parameters } \\
\hline Gender, M/F & $10 / 10$ & $21 / 19$ & - \\
\hline Age, yrs & $61.3(2.17)$ & $61.7(1.73)$ & 0.97 \\
\hline Weight, kg & $72(2.59)$ & $73(3.96)$ & 0.89 \\
\hline $\mathrm{BMl}, \mathrm{kg} \cdot \mathrm{m}^{-2}$ & $25.4(0.76)$ & $26.4(1.26)$ & 0.46 \\
\hline \multicolumn{4}{|l|}{ Pulmonary parameters } \\
\hline $\mathrm{FEV}_{1}, \mathrm{~L}$ & $3.12(0.2)$ & $1.3(0.11)$ & $<0.001$ \\
\hline $\mathrm{FEV}_{1}, \%$ pred & $113.9(3.2)$ & $49.6(3.66)$ & $<0.001$ \\
\hline $\mathrm{FEV}_{1} / \mathrm{FVC}$ & $0.74(0.46)$ & $0.5(0.28)$ & $<0.001$ \\
\hline \multicolumn{4}{|c|}{ Maximal voluntary and electrically evoked quadriceps torque } \\
\hline QMVC, Nm & $150.3(15.7)$ & $99(10.4)$ & $<0.05$ \\
\hline QPt, Nm & $43.1(3.71)$ & $33.5(3.32)$ & $<0.05$ \\
\hline \multicolumn{4}{|c|}{ Voluntary activation parameters } \\
\hline$V A_{\text {peripheral }}$ & $93(1)$ & $93.1(1.32)$ & 0.94 \\
\hline $\mathrm{VA}_{\text {cortical, }} \%$ & $89.8(1.02)$ & $85.7(3.11)$ & 0.26 \\
\hline \multicolumn{4}{|c|}{ Peripheral and spinal parameters } \\
\hline Mmax amplitude, mV & $3.94(0.38)$ & $3.32(0.5)$ & 0.36 \\
\hline Mmax lat, ms & $5.7(0.27)$ & $6.15(0.25)$ & 0.38 \\
\hline Hmax/Mmax & $0.188(0.027)$ & $0.26(0.029)$ & 0.34 \\
\hline Hmax lat, ms & $16.63(0.54)$ & $18.98(0.59)$ & $<0.01$ \\
\hline \multicolumn{4}{|l|}{ Corticospinal parameters } \\
\hline MEP/Mmax & $0.374(0.021)$ & $0.34(0.045)$ & 0.68 \\
\hline $\mathrm{CMCT}, \mathrm{ms}$ & $20.3(0.56)$ & $20.2(0.54)$ & 0.59 \\
\hline $\mathrm{CSP}, \mathrm{ms}$ & $75.6(7.2)$ & $103(4.43)$ & $<0.001$ \\
\hline
\end{tabular}

$B M I$ body mass index, $F E V_{1}$ forced expiratory volume in $1 \mathrm{~s}, F V C$ forced vital capacity, QMVC quadriceps maximal voluntary torque, QPt quadriceps peak twitch, Cortical VA primary motor cortex activation, Mmax amplitude amplitude of the maximal M-wave, Mmax lat latency of the maximal M-wave, Hmax/ Mmax amplitude of the maximal $\mathrm{H}$-wave normalized with respect to the maximal M-wave, Hmax - Mmax lat latency of the maximal $\mathrm{H}$-wave minus the latency of the maximal M-wave, MEP/Mmax amplitude of the maximal motor evoked potential normalized with respect to the maximal M-wave amplitude, CMCT central motor conduction time, CSP cortical silent period. Data are expressed as mean and SE than controls. Moreover, they also exhibited significantly higher Hmax latency $\left(\mathrm{t}_{37}=2.94, p<0.01\right)$ and longer silent period duration $\left(\mathrm{t}_{37}=3.33, p<0.001\right)$ than controls. Interestingly, there were no significant differences between the patients and controls regarding $\mathrm{VA}_{\text {cortical }}$ and MEP/Mmax ( $p=0.26$ and 0.68 , respectively).

\section{Controls and the COPD $\mathrm{MW}$ and $\mathrm{COPD}_{\mathrm{NoMw}}$ groups Participant characteristics}

The participant characteristics are presented in Table 2. The $\mathrm{COPD}_{\mathrm{MW}}$ and $\mathrm{COPD}_{\mathrm{NoMW}}$ groups were composed of $22(\mathrm{M} / \mathrm{F}$ ratio $=10 / 12)$ and 18 patients $(\mathrm{M} / \mathrm{F}=11 / 7)$, respectively. There were no significant differences between the three groups in age, weight or BMI ( $p$-value equal to $0.84,0.67$ and 0.53 , respectively). The two groups of COPD patients did not differ regarding airflow obstruction $\left(\mathrm{FEV}_{1} / \mathrm{FVC}\right)$, obstruction severity $\left(\mathrm{FEV}_{1}\right)$, smoking pack-years and number of comorbidities $(p=$ $0.48,0.62,0.39$ and 0.63 , respectively). In contrast, $\mathrm{PaO}_{2}$ was significantly lower in the $\mathrm{COPD}_{\mathrm{MW}}$ group $(p<0.01)$.

\section{Maximal voluntary and electrically evoked quadriceps torque}

The data are presented in Fig. $3 \mathrm{a}$ and b. Significant effects of group were found for QMVC and QPt $\left(\mathrm{F}_{2,57}=\right.$ 10.73, $p<0.001$ and $\mathrm{F}_{2,57}=4.46, p<0.01$, respectively), which were significantly lower in $\mathrm{COPD}_{\mathrm{MW}}$ compared with the COPD ${ }_{\mathrm{NoMW}}$ and control groups (all post-hoc $p<0.05$ ). Even adjusted for QPt, QMVC remained significantly lower in $\mathrm{COPD}_{\mathrm{MW}}$ (significant effect of group: $\mathrm{F}_{2,56}=6.54, p<0.01$; with both post-hoc $p<0.05$ ). There was no difference in QMVC or QPt between the COPDNoMW and control groups (post-hoc $p=0.14$ and 0.77, respectively). In addition, the percentage of QMVC variance explained by QPt was quite different between healthy controls (adjusted $r^{2}=0.8$ ) and COPD patients (adjusted $\mathrm{r}^{2}=0.65$ and 0.62 for COPD without and with muscle weakness, respectively).

Correlations between QMVC and QPt were statistically significant in all groups ( $\mathrm{r}$ ranged from 0.8 to $0.9 ; \mathrm{p}$ systematically lower than 0.001 ). However, the characteristics of the relationship (slopes and $y$-intercepts) differed according to the group. The $y$-intercept was progressively higher in the $\mathrm{COPD}_{\mathrm{NoMW}}$ (4.8) and $\mathrm{COPD}_{\mathrm{MW}}$ (29.4) groups compared with the control group $(-14)\left(\mathrm{F}_{5,54}\right.$ ranged from 4.53 to 18.9 , $\mathrm{p}$ ranged from 0.001 to 0.04$)$. The slope was not different between the control (3.82) and $\mathrm{COPD}_{\mathrm{NoMW}}(2.8)$ groups $\left(\mathrm{F}_{3,34}=\right.$ $1.82 ; p=0.18$ ) but was significantly lower in the $\mathrm{COPD}_{\mathrm{MW}}$ group (1.6) (comparison versus $\mathrm{COPD}_{\mathrm{NoMW}}$ : $\mathrm{F}_{3,36}=5.25 ; p<0.03$ and versus controls: $\mathrm{F}_{3,38}=20.4 ; p<$ 0.001; see Fig. 4). As a consequence, for equivalent QPt, QMVC tended to be lower in the $\mathrm{COPD}_{\mathrm{MW}}$ group. 
Table 2 Characteristics of controls and patients with $\left(C O P D_{M w}\right)$ or without $\left(C O P D_{N o M w}\right)$ muscle weakness

\begin{tabular}{|c|c|c|c|c|c|c|c|}
\hline & Controls (a) & $\mathrm{COPD}_{\text {NoMW }}(\mathrm{b})$ & $\mathrm{COPD}_{\mathrm{MW}}(\mathrm{c})$ & ANOVA $p$-value & $\begin{array}{l}p \text {-value } \\
\text { a vs b }\end{array}$ & $\begin{array}{l}p \text {-value } \\
\text { a vs c }\end{array}$ & $\begin{array}{l}p \text {-value } \\
\text { b vs C }\end{array}$ \\
\hline Gender, M/F & $10 / 10$ & $11 / 7$ & $10 / 12$ & - & - & - & - \\
\hline Age, yrs & $61.3(2.17)$ & $60.8(2.19)$ & $62.4(1.34)$ & 0.84 & - & - & - \\
\hline Weight, kg & $72(2.59)$ & $75.3(3.42)$ & $71.1(3.94)$ & 0.67 & - & - & - \\
\hline $\mathrm{BMl}, \mathrm{kg} \cdot \mathrm{m}^{-2}$ & $25.4(0.76)$ & $27.3(1.18)$ & $25.8(1.41)$ & 0.53 & - & - & - \\
\hline $\mathrm{FEV}_{1}, \mathrm{~L}$ & $3.12(0.2)$ & $1.34(0.14)$ & $1.23(0.09)$ & $<0.001$ & $<0.001$ & $<0.001$ & 0.62 \\
\hline $\mathrm{FEV}_{1}, \%$ pred & $113.9(3.2)$ & $50.5(4.21)$ & $47.4(3.18)$ & $<0.001$ & $<0.001$ & $<0.001$ & 0.53 \\
\hline $\mathrm{FEV}_{1} / \mathrm{FVC}$ & $0.74(0.11)$ & $0.51(0.32)$ & $0.49(0.27)$ & $<0.001$ & $<0.001$ & $<0.001$ & 0.53 \\
\hline $\mathrm{PaO}_{2}, \mathrm{mmHg}$ & - & $72.2(2.07)$ & $65.4(1.55)$ & - & - & - & $<0.01^{*}$ \\
\hline $\mathrm{PaCO}_{2}, \mathrm{mmHg}$ & - & $40.1(1.59)$ & $40.1(1.1)$ & - & - & - & $0.99 *$ \\
\hline Smoking history (pack-years) & $7.3(12.5)$ & $38.9(26.9)$ & $45.5(24)$ & $<0.001$ & $<0.001$ & $<0.001$ & 0.39 \\
\hline Number of comorbidities & - & $2(1.2)$ & $2.2(1.1)$ & - & - & - & $0.63^{*}$ \\
\hline
\end{tabular}

$\mathrm{BMI}$ Body mass index, $\mathrm{FEV}_{1}$ forced expiratory volume in $1 \mathrm{~s}, \mathrm{FVC}$ forced vital capacity, $\mathrm{PaO}_{2}$ arterial oxygen tension, $\mathrm{PaCO}_{2}$ arterial carbon dioxide tension. Data are mean and SE

(a)

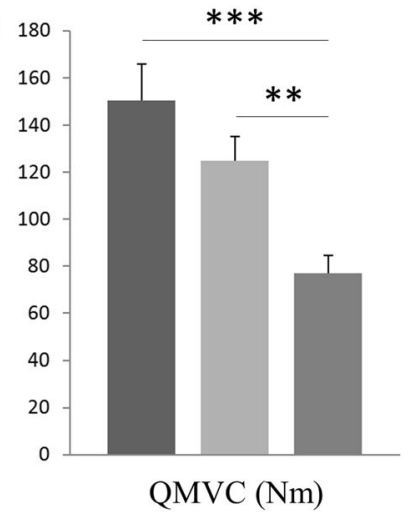

(c)

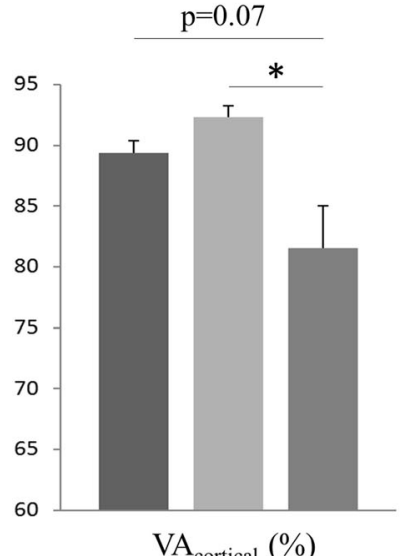

(b)

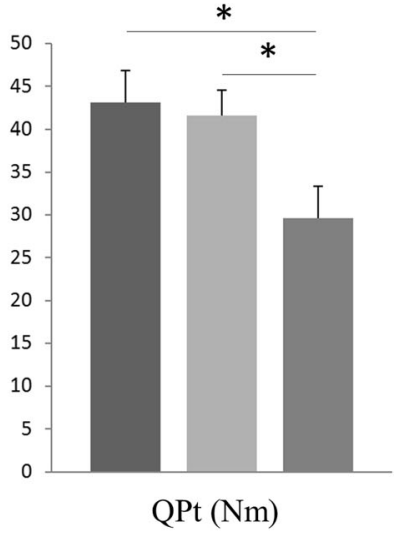

(d)
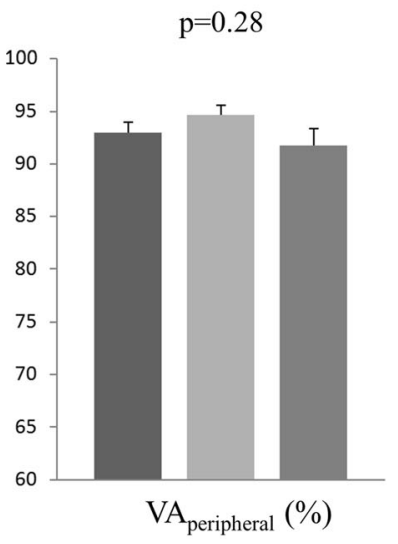

\section{Controls}

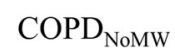

$\mathrm{COPD}_{\mathrm{MW}}$

Fig. 3 a Quadriceps maximal voluntary torque (QMVC), b quadriceps maximal peak twitch (QPt), c voluntary activation estimated with motor cortex transcranial magnetic stimulation during maximal voluntary contractions VA $_{\text {cortical }}(\%)$ ], and $\mathbf{d}$ voluntary activation estimated with twitch interpolation during maximal voluntary contractions $\left[V A_{\text {peripheral }}(\%)\right]$, in healthy controls, patients with COPD and preserved muscle strength $\left(C O P D_{\text {NoMw }}\right)$, and patients with COPD and muscle weakness (COPD $\mathrm{MW}) .{ }^{*},{ }^{* *},{ }^{* * *}: p<0.05, p<0.01$ and $p<0.001$ respectively. Vertical bars represent SE 


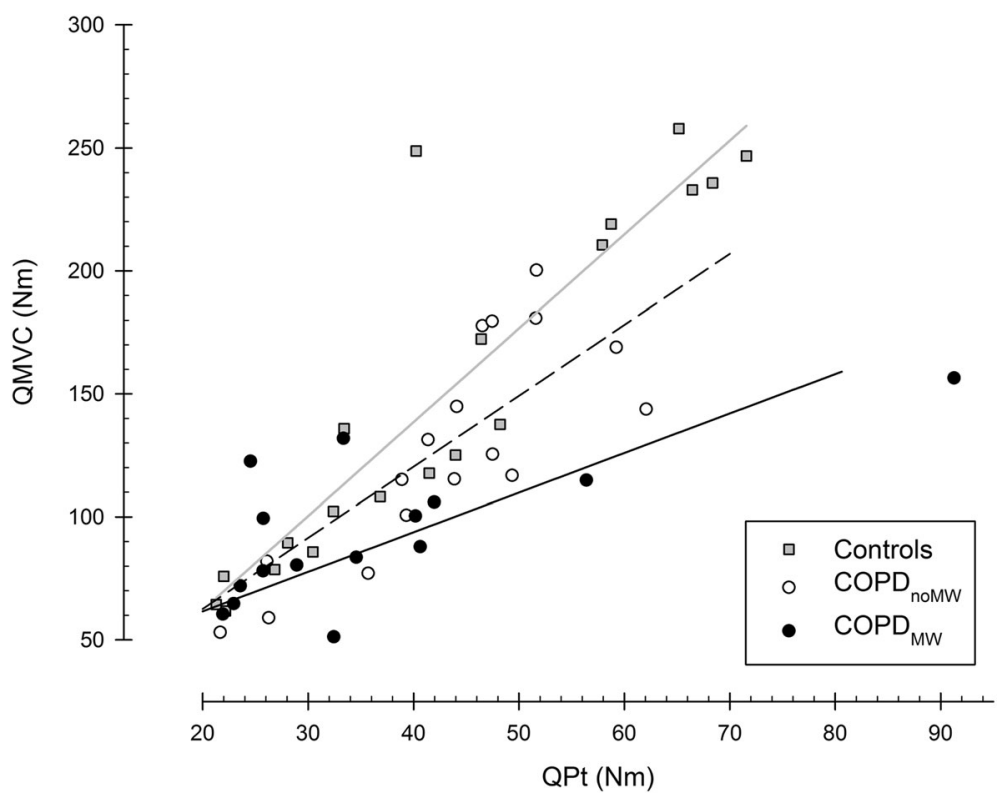

Fig. 4 Slopes and y-intercepts of linear relationship between maximal voluntary quadriceps torque (QMVC) and quadriceps maximal peak twitch $(\mathrm{QPt})$ in healthy controls (gray squares and gray line), COPD patients without muscle weakness (COPD $\mathrm{Nomw}_{\text {) }}$ (empty circles and dotted line), and COPD patients with muscle weakness (COPD $\left.{ }_{\mathrm{MW}}\right)$ (dark circles and dark line)

\section{Peripheral and corticospinal excitability}

The electrophysiological data are presented in Table 3. Sixteen participants (26.6\%: 5 controls, 5 COPD patients with muscle weakness, and 6 COPD patients without muscle weakness) did not exhibit maximal MEP at maximal stimulator output or had no linear relationship between TMS twitches. These participants were excluded from the analyses of data elicited by TMS. Muscular excitability estimated by Mmax amplitude and Mmax latency did not significantly differ between the 3 groups $\left(\mathrm{F}_{2,54}=1.03, p=0.36\right.$ and $\mathrm{F}_{2,56}=0.97, p=0.38$, respectively). Spinal excitability expressed by the Hmax/Mmax ratio was also comparable between the 3 groups $\left(\mathrm{F}_{2,35}=\right.$ 1.44, $p=0.25$ ). A significant effect of group was found for Hmax latency $\left(\mathrm{F}_{2,36}=4.26, p<0.05\right)$, which was significantly higher in the two COPD groups compared with controls (both post-hoc $p<0.05$ ). However, the central motor conduction time was not different between the three groups $\left(\mathrm{F}_{2,35}=1.16, p=0.33\right)$. A significant effect of group was found for corticospinal excitability measured by MEP/Mmax ratio $\left(\mathrm{F}_{2,33}=7.19\right.$, $p<0.01)$, which was significantly lower in the COPD $\mathrm{MW}$ group compared with the $\mathrm{COPD}_{\mathrm{NoMW}}$ and control groups (post-hoc $p<0.05$ and 0.01 , respectively). There

Table 3 Muscle and corticospinal excitability assessment

\begin{tabular}{|c|c|c|c|c|c|c|c|}
\hline & $\begin{array}{l}\text { Controls } \\
\text { (a) }\end{array}$ & $\begin{array}{l}\text { COPD } \\
\text { (b) }\end{array}$ & $\begin{array}{l}\mathrm{COPD}_{\mathrm{MW}} \\
\text { (c) }\end{array}$ & ANOVA $p$-value & $\begin{array}{l}p \text {-value } \\
\text { a vs b }\end{array}$ & $\begin{array}{l}p \text {-value } \\
\text { a vs c }\end{array}$ & $\begin{array}{l}p \text {-value } \\
\text { b vs c }\end{array}$ \\
\hline \multicolumn{8}{|c|}{ Muscle and spinal parameters } \\
\hline Mmax amplitude, mV & $3.94(0.38)$ & $2.96(0.3)$ & $3.58(0.55)$ & 0.36 & - & - & - \\
\hline Mmax lat, ms & $5.7(0.27)$ & $6.21(0.26)$ & $6.11(0.27)$ & 0.38 & - & - & - \\
\hline Hmax/Mmax & $0.188(0.027)$ & $0.25(0.041)$ & $0.265(0.02)$ & 0.25 & - & - & - \\
\hline Hmax lat, ms & $16.63(0.54)$ & $18.86(0.62)$ & $19.17(0.51)$ & $<0.05$ & $<0.05$ & $<0.05$ & 0.77 \\
\hline \multicolumn{8}{|l|}{ Corticospinal parameters } \\
\hline MEP/Mmax & $0.374(0.021)$ & $0.464(0.056)$ & $0.235(0.019)$ & $<0.01$ & 0.22 & $<0.01$ & $<0.05$ \\
\hline $\mathrm{CMCT}, \mathrm{ms}$ & $20.3(0.56)$ & $19.3(0.45)$ & $20.8(0.55)$ & 0.33 & - & - & - \\
\hline $\mathrm{SP}, \mathrm{ms}$ & $75.6(7.2)$ & $108.3(4.55)$ & $98.3(3.79)$ & $<0.01$ & $<0.01$ & $<0.05$ & 0.31 \\
\hline
\end{tabular}

Mmax amplitude amplitude of the maximal M-wave, Mmax lat latency of the maximal M-wave, Hmax/Mmax amplitude of the maximal H-wave normalized with respect to the maximal $M$-wave, Hmax lat latency of the maximal H-wave minus the latency of the maximal M-wave, MEP/Mmax amplitude of the maximal motor evoked potential normalized with respect to the maximal M-wave amplitude, $C M C T$ central motor conduction time, $S P$ silent period duration. Data are mean and SE 
was also a significant effect of group for the silent period duration, which is an indicator of corticospinal inhibition $\left(\mathrm{F}_{2,36}=6.07, p<0.01\right)$. Post-hoc comparisons revealed that the silent period duration was longer in the two COPD groups compared with controls $(p<0.05$ for comparison between $\mathrm{COPD}_{\mathrm{NoMW}}$ and controls, and $p<$ 0.01 for $\mathrm{COPD}_{\mathrm{MW}}$ and controls), without any differences between the two COPD groups $(p=0.31)$.

\section{Voluntary activation}

The mean correlation coefficient of the relationship between twitch-like increment in torque induced by TMS and voluntary torque was $0.979 \pm 0.03$. A significant effect of group was found for $\mathrm{VA}_{\text {cortical }}\left(\mathrm{F}_{2,39}=3.91, p<\right.$ 0.05 ; Fig. 3c) which was significantly lower in $\mathrm{COPD}_{\mathrm{MW}}$ compared with $\operatorname{COPD}_{\mathrm{NoMW}}$ (post hoc $p<0.01$ ). Even adjusted for $\mathrm{PaO}_{2}, \mathrm{VA}_{\text {cortical }}$ remained significantly lower in $\mathrm{COPD}_{\mathrm{MW}}\left(\mathrm{F}_{2,38}=5.87, p<0.05\right)$.

Conversely, $\mathrm{VA}_{\text {peripheral }}$ (Fig. 3d) did not differ between groups $\left(\mathrm{F}_{2,57}=1.32, p=0.28\right)$.

\section{Discussion}

The purpose of the study was to compare corticospinal and muscle functions in COPD patients with and without peripheral muscle weakness. Compared with patients with preserved muscle strength, patients with muscle weakness exhibited lower $\mathrm{VA}_{\text {cortical }}$ during maximal voluntary contractions associated with a lower MEP/Mmax ratio. Conversely, no differences were found between patients with and without muscle weakness regarding silent period duration, central motor conduction time or M-wave properties.

Peripheral muscle alterations were assessed by QPt (non-voluntary contraction, reflecting both muscle structure and function) in this study. We found lower QPt in patients with muscle weakness compared with healthy controls and patients with normal muscle strength. These findings concur with those of a previous study highlighting the implication of peripheral alterations in the poor quadriceps strength of the weakest COPD patients [21]. However, several clues are in agreement with a moderate role. First, when QPt was included as a covariate in ANCOVA analysis, the maximal voluntary contraction (QMVC) remained significantly lower in $C O P D_{M W}$. Second, the percentage of QMVC variance explained by QPt was sharply lower in COPD patients with muscle weakness $(62 \%)$ than in control subjects (80\%). These data indicate that peripheral muscle alterations are less decisive in maximal force production in COPD patients with muscle weakness. Third, as shown in Fig. 4, the slope between QMVC and QPt was significantly lower in patients with muscle weakness compared with patients without muscle weakness and healthy controls. In other words, for a given
QPt, the patients with muscle weakness tended to exhibit lower QMVC than the other groups. Collectively, these data point to the existence of an additional nonmuscular limiting factor in COPD muscle weakness. The reduced neural excitability and the voluntary activation deficit that we found in parallel in the patients with muscle weakness are relevant explanations for the unexplained part of COPD muscle weakness.

The existence of a voluntary activation deficit has been a controversial issue in COPD. A first consideration is the relevance of the twitch interpolation method during maximal voluntary contractions to assess voluntary activation [11-14]. In the current study, we provided an additional estimation of voluntary activation by directly stimulating at the motor cortex level. We observed lower $\mathrm{VA}_{\text {cortical }}$ in the patients with muscle weakness, despite no $\mathrm{VA}_{\text {peripheral }}$ differences. This is not the first time that a mismatch between $\mathrm{VA}_{\text {cortical }}$ and $\mathrm{VA}_{\text {peripheral }}$ has been reported over the knee extensor muscles [42-44]. The absence or relatively poor $\mathrm{VA}_{\text {peripheral }}$ changes compared with the $\mathrm{VA}_{\text {cortical }}$ changes has been explained by a lack of sensitivity of the twitch interpolation method [42, 44]. Indeed, this method cannot provide a direct indication of the amount of neural drive reaching the muscle because the assessment takes place at the muscle level $[45,46]$. Moreover, the twitch interpolation method presents a nonlinear relationship at high force levels, such that changes in the voluntary force elicit minimal changes in the superimposed twitch size [16-18]. For example, in a previous study, a gain of $5.7 \%$ in $\mathrm{VA}_{\text {peripheral }}$ induced an average $20.4 \%$ increase in QMVC [18]. Another consideration is that voluntary activation and neural activity have previously been assessed in COPD patients as a whole without discriminating the patients with and without muscle weakness [11-15, 47]. At first glance, the absence of a significant difference in $\mathrm{VA}_{\text {cortical }}$ between the controls and the patients as a whole (Table 1) might have led us to conclude that an activation deficit was probably not involved in the reduced quadriceps strength in COPD. However, the patients with quadriceps muscle weakness exhibited a significantly lower $\mathrm{VA}_{\text {cortical }}$ compared with patients with preserved quadriceps strength. This result suggests that in many patients, reduced motor cortex activation is involved in quadriceps muscle weakness. A lower MEP/Mmax ratio was also noted in the patients with quadriceps muscle weakness. Changes in MEP/Mmax can reflect changes in spinal or cortical excitability [48]. In the current study, spinal excitability (Hmax/Mmax) did not differ between groups. Thus, the lower MEP/Mmax ratio suggests reduced motor cortex excitability in the patients with quadriceps muscle weakness, which supports the hypothesis of reduced voluntary activation from the motor cortex in these patients. Importantly, the only difference in pulmonary and blood gas data between the two groups 
of patients concerned the resting $\mathrm{PaO}_{2}$ levels, which were significantly lower in the weak patients. To check that the $\mathrm{VA}_{\text {cortical }}$ alteration was not biased by hypoxemia differences, we performed an ANCOVA with $\mathrm{PaO}_{2}$ as covariate. $\mathrm{VA}_{\text {cortical }}$ remained significantly lower in the patients with muscle weakness $(\mathrm{F}=5.87, p<0.05)$. Furthermore, any oxygen desaturation during the quadriceps contraction of the study protocol was unlikely, since in a previous study where $\mathrm{SpO} 2$ was measured during similar efforts, the mean variation of $\mathrm{SpO}_{2}$ was only 0.01 and non-significant [15]. Consequently, the significantly lower $\mathrm{VA}_{\text {cortical }}$ in the weak patients can be considered as a consistent result.

In this study, we also aimed to assess the mechanisms potentially involved in the decreased voluntary activation in COPD. Silent period duration is thought to reflect the level of corticospinal inhibition [49]. In accordance with a previous study, we found a lengthened silent period in COPD patients compared with healthy controls [50]. However, the increased silent period duration in both groups of COPD patients regardless of muscle weakness necessarily indicates that higher corticospinal inhibition is not responsible for most of the observed loss of voluntary strength in the patients with muscle weakness.

Peripheral neuropathy has been widely described in COPD and has mainly been characterized by lower peripheral nerve conduction velocities and lower Mmax amplitude [25-28]. In the current study, we found no differences regarding Mmax amplitude and latency between the two groups of patients and the healthy controls. These results suggest the preservation of neuromuscular transmission at the motor neuron and/or the motor plate level in COPD. This is also supported by the comparable central motor conduction time (corresponding to the MEP latency) between groups, which depends on both motor neuron and corticospinal conduction velocities. Conversely, we noted lengthened spinal reflex latency (Hmax latency) in the patients with COPD compared with healthy controls, regardless of muscle weakness. The alteration in Hmax latency without any central motor conduction time changes could be explained by impairment at the Ia afferent pathways, as the other pathways traveled by the H-reflex are the same as those traveled by the MEP. Therefore, these results suggest a selective alteration in the quadriceps Ia afferent pathways in the patients with COPD, which is in agreement with previous studies reporting greater impairment in the fascicles of sensory nerves than in motor nerves in these patients [25]. Moreover, the lengthened Hmax latency regardless of muscle weakness also indicates that the alterations in the sensory nerve fascicles are not responsible for most of the observed loss of voluntary strength in the patients with muscle weakness.

In sum, higher corticospinal inhibition and impaired neuromuscular transmission are unlikely to be involved in the reduced quadriceps strength of the patients with muscle weakness. The most likely explanatory mechanism of the neural component of peripheral muscle weakness, and thus for reduced voluntary activation, is decreased excitation from the brain, which is supported by the observation of lower gray matter density in the motor cortex (precentral gyrus) in COPD [22].

Although not a direct objective of the current study, our results may provide some new insights in the potential trigger for brain impairment in COPD. Several etiological factors have been advanced to explain brain impairment in COPD, the most important of which are cerebral vascular disease, inflammation, oxidative stress, smoking, hypoxemia and non-rapid eye movement (NREM) sleep desaturation [51, 52]. First, we did not find any differences in smoking history between the two groups of COPD patients. Thus, a major implication of cigarette smoke in the reported brain alterations is unlikely. Furthermore, without being able to definitely discard the implication of hypoxemia, our results show that the reduced cortical activation was independent of $\mathrm{PaO}_{2}$ levels.

Beyond mechanistic perspectives, the results of the study open new horizons for muscle weakness management in patients with COPD. Indeed, some specific interventions are known to promote neural adaptations such as eccentric exercise [53], neuromodulation [54] or electrical stimulation strength training [55]. These interventions might help to improve responses to rehabilitation in COPD patients with muscle weakness from cortical origin. This is particularly relevant for efforts to improve adaptations to pulmonary rehabilitation programs, given the rate of patients who respond poorly to the classical programs [56].

Methodological considerations.

We used a figure-of-eight coil to stimulate the leg motor cortex. Although a double-cone coil is more conventional to stimulate the leg motor cortex as the magnetic stimulus penetrates less deeply for a given intensity [57], the figureof-eight coil takes the advantage to be more focal [58], and there is no clear rationale to give priority to one over the other in the literature [57]. Apart from these considerations, some strict procedures helped us to increase the validity of the data provided by the figure-of-eight coil. First, to avoid potentially suboptimal output, we used a higher intensity (mean stimulator output was 96\%) than the intensities usually reported with a double-cone coil $(\approx 60-70 \%)$. We also excluded from the TMS data analyses the participants with no MEP plateau before reaching the maximal stimulator output (26.6\%). This rate of exclusion was not much higher than in previous studies. For example, in younger healthy subjects, a rate of exclusion between 15 and 20\% was reported [59, 60]. In addition, the TMS voluntary activation data were quite consistent with the literature data. In the current study, the mean TMS voluntary activation using a figure-of-eight coil was $89 \%$ 
in the healthy elderly controls and $92 \%$ in the patients without muscle weakness. In a previous study using a double-cone coil on the same muscle group and on a similar population (healthy elderly), mean TMS voluntary activation was $90 \%$ [61].

Another limitation was that the antagonist biceps femoris Mmax could not be measured in the study. The minimization of antagonist muscle activity during TMS is important to provide appropriate TMS data [41]. However, the stimulation of the sciatic nerve to elicit biceps femoris Mmax is very challenging for technical and ethical reasons. First, the site of stimulation is surrounded by large muscles, which makes it difficult to avoid stimulating a muscle directly. Furthermore, to evoke Mmax on the biceps femoris, a very high stimulation intensity on the sciatic nerve (over $600 \mathrm{~mA}$ ) is needed [19]. This intensity is more than six times higher than that used for stimulating the femoral nerve in the current study. The use of such painful intensity is inconceivable in patients with chronic illness. Nevertheless, several strategies were employed to minimize antagonist activity during TMS. First, the skin impedance was rather null in both groups $(<5 \mathrm{k} \Omega)$. Second, we systematically checked to obtain the smallest possible response on the antagonist muscle by moving the coil by small amounts. Last, the study included a control group and, given the two aforementioned procedures, there is no reason to believe that the antagonist was more activated in one group than the others.

\section{Conclusions}

In addition to peripheral muscle alterations (lower QPt), patients with COPD and muscle weakness exhibit lower motor cortex excitability during maximal voluntary contractions and, logically, lower voluntary activation from the motor cortex. A decrease in neural excitation is the most likely mechanism responsible for the lower motor cortex activation in these patients. This study provides evidence of specific cortical implication in COPD with muscle weakness. This may have important implications for pulmonary rehabilitation and it could potentially explain why some patients exhibit low responsiveness to retraining programs. Thus, the question of specific interventions in patients with muscle weakness of cortical origin needs to be addressed. Further, future studies and research must be carried out on the factors responsible for motor cortex impairment in COPD and the possible therapeutic targets.

\section{Abbreviations}

ANCOVA: Analysis of covariance; ANOVA: Analysis of variance; COPD: Chronic obstructive pulmonary disease; $C_{\mathrm{MW}}$ : $\mathrm{COPD}$ patients with quadriceps muscle weakness; COPD nomw: COPD patients with quadriceps strenght; EMG: Electromyography; $\mathrm{FEV}_{1}$ : Forced expiratory volume in the 1st second; FVC: Forced vital capacity; Hmax: Maximal H-reflex; IMmax: Intensity at which Mmax is obtained; MEP: Motor evoked potential; Mmax: Maximal compound muscle action potential; QMVC: Isometric maximal quadriceps torque; QPt: Quadriceps Peak twitch; TMS: Transcranial magnetic stimulation;
VA cortical: Voluntary activation estimated by TMS; VA peripheral: Voluntary activation estimated by peripheral nerve stimulation

\section{Acknowledgments}

The authors want to thank C. Stott Carmeni for English revision of the manuscript.

\section{Authors' contributions}

$\mathrm{FA}, \mathrm{NH}, \mathrm{DB}$ and $\mathrm{AV}$ conceived and designed the study. FA, ET and NO performed the data acquisition. FA, NH, ET and $\mathrm{AV}$ analyzed the data, and $\mathrm{FA}, \mathrm{NH}, \mathrm{ET}, \mathrm{DB}$ and $\mathrm{AV}$ interpreted the data. FA drafted the manuscript. All authors have read and approved the final manuscript.

\section{Funding}

François ALEXANDRE was funded by the French National Agency for Research and Technology (CIFRE N²011/0894). The body funding was not involved in the design of the study and collection, analysis, and interpretation of data and in writing the manuscript.

Availability of data and materials

The datasets that were used and analyzed during the current study are available from the corresponding author on reasonable request.

\section{Ethics approval and consent to participate}

The study protocol was approved by the local ethics committee (Comité de Protection des Personnes Sud-Est VI, Clermont-Ferrand, number AU980). All participants provided informed written consent prior to the start of the study.

\section{Consent for publication}

Not applicable.

\section{Competing interests}

The authors declare that they have no competing interests.

Received: 10 May 2019 Accepted: 24 December 2019

Published online: 03 January 2020

References

1. Barreiro E, Gea J. Respiratory and limb muscle dysfunction in COPD. COPD: J Chron Obstruct Pulmon Dis. 2015;12(4):413-26.

2. Bernard S, LeBLANC P, Whittom F, Carrier G, Jobin J, Belleau R, et al. Peripheral muscle weakness in patients with chronic obstructive pulmonary disease. Am J Respir Crit Care Med. 1998;158(2):629-34.

3. Gosselink R, Troosters T, Decramer M. Peripheral muscle weakness contributes to exercise limitation in COPD. Am J Respir Crit Care Med. 1996:153(3):976-80.

4. Locke E, Thielke S, Diehr P, Wilsdon AG, Graham Barr R, Hansel N, et al. Effects of respiratory and non-respiratory factors on disability among older adults with airway obstruction: the cardiovascular health study. COPD: J Chron Obstruct Pulmon Dis. 2013;10(5):588-96.

5. Swallow EB, Reyes D, Hopkinson NS, Man WD, Porcher R, Cetti EJ, et al. Quadriceps strength predicts mortality in patients with moderate to severe chronic obstructive pulmonary disease. Thorax. 2007:62(2):115-20.

6. Clark BC, Manini TM. Sarcopenia $\neq$ dynapenia. J Gerontol Ser A Biol Med Sci. 2008;63(8):829-34

7. Clark BC, Taylor JL, Hong SL, Law TD, Russ DW. Weaker seniors exhibit motor cortex hypoexcitability and impairments in voluntary activation. Gerontol Series A: Biomedical Sciences and Medical Sciences. 2015;70(9): $1112-9$

8. Debigare R, Cote C, Hould F, LeBlanc P, Maltais F. In vitro and in vivo contractile properties of the vastus lateralis muscle in males with COPD. Eur Respir J. 2003:21(2):273-8.

9. Maddocks M, Shrikrishna D, Vitoriano S, Natanek SA, Tanner RJ, Hart N, et al. Skeletal muscle adiposity is associated with physical activity, exercise capacity and fibre shift in COPD. Eur Respir J. 2014;44(5):1188-98.

10. Natanek SA, Gosker HR, Slot IG, Marsh GS, Hopkinson NS, Man WDC, et al. Heterogeneity of quadriceps muscle phenotype in chronic obstructive pulmonary disease (COPD); implications for stratified medicine? Muscle Nerve. 2013;48(4):488-97. 
11. Maddocks M, Jones M, Snell T, Connolly B, Wolf-Linder S, Moxham J, et al. Ankle dorsiflexor muscle size, composition and force with ageing and chronic obstructive pulmonary disease. Exp Physiol. 2014;99(8):1078-88.

12. Mador MJ, Deniz O, Aggarwal A, Kufel TJ. Quadriceps fatigability after single muscle exercise in patients with chronic obstructive pulmonary disease. Am J Respir Crit Care Med. 2003;168(1):102-8.

13. Seymour JM, Ward K, Raffique A, Steier JS, Sidhu PS, Polkey MI, et al. Quadriceps and ankle dorsiflexor strength in chronic obstructive pulmonary disease. Muscle Nerve. 2012;46(4):548-54.

14. Vivodtzev I, Flore P, Lévy P, Wuyam B. Voluntary activation during knee extensions in severely deconditioned patients with chronic obstructive pulmonary disease: benefit of endurance training. Muscle Nerve. 2008;37(1): 27-35.

15. Alexandre F, Heraud N, Oliver N, Varray A. Cortical implication in lower voluntary muscle force production in non-hypoxemic COPD patients. PLoS One. 2014;9(6):e100961

16. de Hann A, Gerrits K, de Ruiter C. Counterpoint: the interpolated twitch does not provide a valid measure of the voluntary activation of muscle. J Appl Physiol. 2009;107:355-7.

17. Herbert R, Gandevia S. Twitch interpolation in human muscles: mechanisms and implications for measurement of voluntary activation. J Neurophysiol. 1999;82(5):2271-83.

18. Kooistra R, De Ruiter C, De Haan A. Conventionally assessed voluntary activation does not represent relative voluntary torque production. Eur J Appl Physiol. 2007;100(3):309-20.

19. Sidhu SK, Bentley DJ, Carroll TJ. Cortical voluntary activation of the human knee extensors can be reliably estimated using transcranial magnetic stimulation. Muscle Nerve. 2009;39(2):186-96.

20. Jones SE, Maddocks M, Kon SS, Canavan JL, Nolan CM, Clark AL, et al. Sarcopenia in COPD: prevalence, clinical correlates and response to pulmonary rehabilitation. Thorax. 2015;70(3):213-8.

21. Seymour J, Spruit M, Hopkinson N, Natanek S, Man W-C, Jackson A, et al. The prevalence of quadriceps weakness in COPD and the relationship with disease severity. Eur Respir J. 2010;36(1):81-8.

22. Zhang $H$, Wang $X$, Lin J, Sun $Y$, Huang $Y$, Yang T, et al. Grey and white matter abnormalities in chronic obstructive pulmonary disease: a casecontrol study. BMJ Open. 2012;2(2):e000844.

23. Zhang $H$, Wang $X$, Lin J, Sun $Y$, Huang $Y$, Yang $T$, et al. Reduced regional gray matter volume in patients with chronic obstructive pulmonary disease: a voxel-based morphometry study. Am J Neuroradiol. 2013;34(2):334-9.

24. Ryu C-W, Jahng G-H, Choi CW, Rhee HY, Kim M-J, Kim SM, et al. Microstructural change of the brain in chronic obstructive pulmonary disease: a voxel-based investigation by MRI. COPD: J Chron Obstruct Pulmon Dis. 2013;10(3):357-66.

25. Agrawal D, Vohra R, Gupta P, Sood S. Subclinical peripheral neuropathy in stable middle-aged patients with chronic obstructive pulmonary disease. Singap Med J. 2007:48(10):887.

26. Jann S, Gatti A, Crespi S, Rolo J, Beretta S. Peripheral neuropathy in chronic respiratory insufficiency. J Peripher Nerv Syst. 1998;3(1):69-74

27. Oncel C, Baser S, Cam M, Akdağ B, Taspinar B, Evyapan F. Peripheral neuropathy in chronic obstructive pulmonary disease. COPD. 2010;7(1):11-6.

28. Valli G, Barbieri S, Sergi P, Fayoumi Z, Berardinelli P. Evidence of motor neuron involvement in chronic respiratory insufficiency. J Neurol Neurosurg Psychiatry. 1984;47(10):1117-21.

29. Darques JL, Decherchi $P$, Jammes Y. Mechanisms of fatigue-induced activation of group IV muscle afferents: the roles played by lactic acid and inflammatory mediators. Neurosci Lett. 1998;257(2):109-12.

30. Taylor JL, Amann M, Duchateau J, Meeusen R, Rice CL. Neural contributions to muscle fatigue: from the brain to the muscle and back again. Med Sci Sports Exerc. 2016;48(11):2294.

31. Eagan TM, Ueland T, Wagner PD, Hardie JA, Mollnes TE, Damås JK, et al. Systemic inflammatory markers in COPD: results from the Bergen COPD cohort study. Eur Respir J. 2010;35(3):540-8.

32. Gosker HR, Zeegers MP, Wouters EF, Schols AM. Muscle fibre type shifting in the vastus lateralis of patients with COPD is associated with disease severity: a systematic review and meta-analysis. Thorax. 2007;62(11):944-9.

33. The National Isometric Muscle Strength (NIMS) Database Consortium. Muscular weakness assessment: use of normal isometric strength data. Arch Phys Med Rehabil 1996;77:1251-1255.

34. Sillen MJ, Franssen FM, Delbressine JM, Vaes AW, Wouters EF, Spruit MA Efficacy of lower-limb muscle training modalities in severely dyspnoeic individuals with COPD and quadriceps muscle weakness: results from the DICES trial. Thorax. 2014;69(6):525-31.

35. From the Global strategy for the diagnosis, management, and prevention of COPD. Global Initiative for Chronic Obstructive Lung Disease (GOLD). 2017: Available from: http://www.goldcopd.org/.

36. Quanjer P, Tammeling G, Cotes J, Pedersen O, Perlin R, Yernault J. Lung volumes and forced ventilatory flows. Report working party. Standardization of lung function test European Community for steel and oral official statement of the European Respiratory Society. Eur Respir J. 1993;6(5):40.

37. Hoffman M, Schrader J, Applegate T, Koceja D. Unilateral postural control of the functionally dominant and nondominant extremities of healthy subjects. J Athl Train. 1998;33(4):319.

38. Duchateau J. Comments on point: counterpoint: the interpolated twitch does/does not provide a valid measure of the voluntary activation of muscle. J Appl Physiol. 2009;107(1):361.

39. Panizza M, Nilsson J, Roth BJ, Basser PJ, Hallett M. Relevance of stimulus duration for activation of motor and sensory fibers: implications for the study of H-reflexes and magnetic stimulation. Electroencephalography and Clinical Neurophysiology/Evoked Potentials Section. 1992;85(1):22-9.

40. Temesi J, Gruet M, Rupp T, Verges S, Millet GY. Resting and active motor thresholds versus stimulus-response curves to determine transcranial magnetic stimulation intensity in quadriceps femoris. J Neuroengineering Rehab. 2014;11(1):40.

41. Todd G, Taylor JL, Gandevia SC. Measurement of voluntary activation based on transcranial magnetic stimulation over the motor cortex. J Appl Physiol. 2016;121(3):678-86.

42. Goodall S, González-Alonso J, Ali L, Ross EZ, Romer LM. Supraspinal fatique after normoxic and hypoxic exercise in humans. J Physiol. 2012;590(11): 2767-82.

43. Goodall S, Ross EZ, Romer LM. Effect of graded hypoxia on supraspinal contributions to fatigue with unilateral knee-extensor contractions. J Appl Physiol. 2010;109(6):1842-51.

44. Sidhu SK, Bentley DJ, Carroll TJ. Locomotor exercise induces long-lasting impairments in the capacity of the human motor cortex to voluntarily activate knee extensor muscles. J Appl Physiol. 2009;106(2):556-65.

45. Jakobi JM. Twitch interpolation a valid measure with misinterpreted meaning. J Appl Physiol (Bethesda, Md: 1985). 2009;107(1):362.

46. Taylor JL. Point: counterpoint: the interpolated twitch does/does not provide a valid measure of the voluntary activation of muscle. J Appl Physiol. 2009;107(1):354-5.

47. Ju C, Chen R. Factors associated with impairment of quadriceps muscle function in chinese patients with chronic obstructive pulmonary disease. PLoS One. 2014;9(2):e84167.

48. Fryer $\mathrm{G}$, Pearce AJ. The effect of lumbosacral manipulation on corticospinal and spinal reflex excitability on asymptomatic participants. J Manip Physiol Ther. 2012;35(2):86-93.

49. Inghilleri M, Berardelli A, Cruccu G, Manfredi M. Silent period evoked by transcranial stimulation of the human cortex and cervicomedullary junction. J Physiol. 1993:466(1):521-34.

50. Mohamed-Hussein A, Hamed S, Abdel-Hakim N. Cerebral cortical dysfunction in chronic obstructive pulmonary disease: role of transcranial magnetic stimulation. Int J Tuberculosis Lung Dis. 2007;11(5):515-21.

51. Alexandre F, Heraud N, Varray A. Is nocturnal desaturation a trigger for neuronal damage in chronic obstructive pulmonary disease? Med Hypotheses. 2015;84(1):25-30.

52. Dodd JW, Chung AW, van den Broek MD, Barrick TR, Charlton RA, Jones PW. Brain structure and function in chronic obstructive pulmonary disease: a multimodal cranial magnetic resonance imaging study. Am J Respir Crit Care Med. 2012;186(3):240-5.

53. Hedayatpour N, Falla D. Physiological and neural adaptations to eccentric exercise: mechanisms and considerations for training. Biomed Res Int. 2015; 2015:193741.

54. Tanaka S, Takeda K, Otaka Y, Kita K, Osu R, Honda M, et al. Single session of transcranial direct current stimulation transiently increases knee extensor force in patients with hemiparetic stroke. Neurorehabil Neural Repair. 2011; 25(6):565-9.

55. Hortobágyi T, Maffiuletti NA. Neural adaptations to electrical stimulation strength training. Eur J Appl Physiol. 2011;111(10):2439-49.

56. Spruit MA, Augustin IM, Vanfleteren L, Janssen DJ, Gaffron S, Pennings H-J, et al. Differential response to pulmonary rehabilitation in COPD: multidimensional profiling. Eur Respir J. 2015;46(6):1625-35 ERJ-00350-2015. 
57. Kesar TM, Stinear JW, Wolf SL. The use of transcranial magnetic stimulation to evaluate cortical excitability of lower limb musculature: challenges and opportunities. Restor Neurol Neurosci. 2018;36(3):333-48.

58. Deng Z-D, Lisanby SH, Peterchev AV. Electric field depth-focality tradeoff in transcranial magnetic stimulation: simulation comparison of 50 coil designs. Brain stimulation. 2013;6(1):1-13.

59. Thomas K, Goodall S, Stone M, Howatson G, Gibson ASC, Ansley L. Central and peripheral fatigue in male cyclists after 4-, 20-, and 40-km time trials. Med Sci Sports Exerc. 2015;47(3):537-46.

60. van Duinen H, Gandevia SC, Taylor JL. Voluntary activation of the different compartments of the flexor digitorum profundus. J Neurophysiol. 2010; 104(6):3213-21.

61. Souron R, Besson T, Lapole T, Millet GY. Neural adaptations in quadriceps muscle after 4 weeks of local vibration training in young versus older subjects. Appl Physiol Nutr Metab. 2017;43(5):427-36.

\section{Publisher's Note}

Springer Nature remains neutral with regard to jurisdictional claims in published maps and institutional affiliations.

Ready to submit your research? Choose BMC and benefit from:

- fast, convenient online submission

- thorough peer review by experienced researchers in your field

- rapid publication on acceptance

- support for research data, including large and complex data types

- gold Open Access which fosters wider collaboration and increased citations

- maximum visibility for your research: over $100 \mathrm{M}$ website views per year

At BMC, research is always in progress.

Learn more biomedcentral.com/submissions 